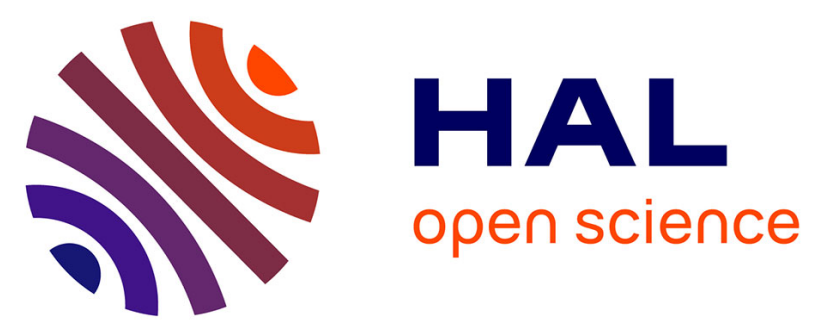

\title{
Immunisation par les chimiothérapies anti-cancéreuses : le point en 2012
}

Dalil Hannani, Laetitia Aymeric, Oliver Kepp, Isabelle Martins, Guido Kroemer, Laurence Zitvogel

\section{- To cite this version:}

Dalil Hannani, Laetitia Aymeric, Oliver Kepp, Isabelle Martins, Guido Kroemer, et al.. Immunisation par les chimiothérapies anti-cancéreuses: le point en 2012. Bulletin de l'Académie Nationale de Médecine, 2012, 196, pp.1075 - 1086. hal-02047413

\section{HAL Id: hal-02047413 \\ https://hal.science/hal-02047413}

Submitted on 17 Dec 2020

HAL is a multi-disciplinary open access archive for the deposit and dissemination of scientific research documents, whether they are published or not. The documents may come from teaching and research institutions in France or abroad, or from public or private research centers.
L'archive ouverte pluridisciplinaire HAL, est destinée au dépôt et à la diffusion de documents scientifiques de niveau recherche, publiés ou non, émanant des établissements d'enseignement et de recherche français ou étrangers, des laboratoires publics ou privés. 


\title{
« Immunisation par les chimiothérapies anticancéreuses : le point en 2012 »
}

\author{
MOTS-CLES : APOPTOSE, CHIMIOTHERAPIE, IMMUNITE INNEE, CELLULES DENDRITIQUES
}

\author{
Immunization through anticancer chemotherapy \\ KEYWORDS: APOPTOSIS, CHEMOTHERAPY, INNATE IMMUNITY, DENDRITIC CELLS
}
Dalil HANNANI ${ }^{1,2,3}$, Laetitia AYMERIC ${ }^{1,2,3}$, Oliver KEPP ${ }^{1,2,4}$, Isabelle MARTINS ${ }^{1,2,4}$, Guido $\mathrm{KROEMER}^{4,5,6,7,8}$, et Laurence ZITVOGEL ${ }^{1,2,3,9}$

\author{
${ }^{1}$ Institut Gustave Roussy, F-94805 Villejuif, France \\ ${ }^{2}$ Faculté Paris Sud-Université Paris XI, France \\ Villejuif, France \\ ${ }^{5}$ Metabolomics Platform, Institut Gustave Roussy, Villejuif, France; \\ ${ }^{6}$ Centre de Recherche des Cordeliers, Paris, France; \\ ${ }^{7}$ Pôle de Biologie, Hôpital Européen Georges Pompidou, AP-HP, Paris, France; \\ ${ }^{8}$ Université Paris Descartes, Paris 5, Paris, France \\ ${ }^{9}$ Centre d'Investigations Clinique BT507 CIC BT507, F-94805 Villejuif, France
}

${ }^{3}$ Institut National de la Santé et de la Recherche Médicale, U1015 "Immunologie et immunothérapie des tumeurs", F-94805

${ }^{4}$ Institut National de la Santé et de la Recherche Médicale, U848 "Apoptosis, Cancer and Immunity", F-94805 Villejuif, France

\section{RESUME}

Le mécanisme d'action supposé de la plupart des traitements anticancéreux résulte d'une action cytotoxique directe sur les cellules tumorales. Dans un numéro de 2008 du Bulletin de l'Académie Nationale de Médecine, nous proposions une théorie alternative ou complémentaire fondée sur nos résultats expérimentaux selon laquelle le système immunitaire contribuerait à l'efficacité de certaines chimiothérapies et radiothérapie antitumorales. Notre travail a montré certains des mécanismes moléculaires de l'immunogénicité de la mort cellulaire induite par les agents thérapeutiques classiques. Ainsi, les signaux échangés entre la tumeur mourante et le système immunitaire conduiraient-ils à l'activation des lymphocytes $T$ par les cellules dendritiques (CD). Nous avons décrypté l'importance du stress du réticulum endoplasmique qui permet la relocalisation de la calréticuline à la membrane plasmique de la cellule tumorale, contribuant ainsi à la phagocytose des corps apoptotiques par les CD. Nous rapportons ici qu'un second stress prémortem, l'autophagie, doit être déclenché pour permettre l'émission de l'ATP dans le milieu extracellulaire, générateur du recrutement des cellules présentatrices d'antigènes et de l'activation de l'inflammasome des CD pour la sécrétion d'IL-1 $\beta$ et la polarisation des lymphocytes $T$ effecteurs. L'architecture de la tumeur se modifie après chimiothérapie efficace, permettant l'accumulation intratumorale séquentielle d'acteurs de l'immunité innée et acquise qui agiront de concert pour l'élimination des cellules tumorales. Ces résultats permettent de générer de nouveaux facteurs prédictifs de réponse aux anthracyclines et de nouvelles stratégies d'immunochimiothérapie.

\section{SUMMARY}

Most of the anticancer agents of the oncological armamentarium supposingly mediate their efficacy through a direct killing (apoptotic and necrotic) activity on tumor, stromal and endothelial cells. In one of the 2008 issues of The Bulletin de l'Académie Nationale de Médecine, we described an alternative or complementary theory whereby the immune system participates to the antitumor effects mediated by some of the chemo-or radio-therapy regimen by promoting an immunogenic cell death pathway. We unraveled the critical importance of two pre-mortem stresses that will condition the immunogenicity of dying tumor cells. First, an ER stress response culminating in calreticuline exposure at the tumor cell surface is mandatory for the uptake and efficient phagocytosis of apoptotic bodies by dendritic cells. Secondly, most of the components of the autophagy machinery are involved in the release of ATP by dying tumors. ATP secretion is crucial for the recruitment of inflammatory phagocytes and antigen presenting cells, as well as for triggering the inflamasome plateforme that causes $I L-1 \beta$ release and $C D 8^{+} T$ cell polarization. Therefore, the architecture of the tumor microenvironemnt will change post-chemotherapy, favoring the sequential accumulation of a series of innate and cognate effectors that will act in a coordinated fashion to promote tumor eradication. These findings will allow to define immune predictors of response to conventional anticancer treatments and to design innovative combinatorial immunochemotherapy regimen. 


\section{Introduction}

\section{Mort cellulaire par chimiothérapie : immunité ou tolérance?}

Le travail de notre équipe ces huit dernières années a permis de démontrer la contribution du système immunitaire à l'efficacité de certaines chimiothérapies ou radiothérapie.

Dans divers modèles de tumeurs transplantées (sarcomes, cancers mammaires, cancers coliques, thymomes, ostéosarcomes) dans divers fonds génétiques de souris mais aussi dans des modèles de sarcomes spontanés induits par le méthylcholanthrène, nous avons montré que l'absence de lymphocytes (plus particulièrement les $\mathrm{T} C \mathrm{CD} 4 \alpha \beta^{+}, \mathrm{T} \mathrm{CD}^{+} \alpha \beta, \gamma \delta^{+} \mathrm{T}$ ) abolit l'efficacité des traitements cytotoxiques. De plus, les cytokines de l'immunité innée (comme IL-17, IL-1 $\beta$ et leurs récepteurs respectifs IL-17RA, IL-1R1) et de l'immunité acquise (IFN $\gamma$, IFNARII) sont indispensables au succès des cytotoxiques antitumoraux. Cette démonstration a été apportée grâce aux animaux génétiquement déficients pour l'expression de la recombinase RAG, IFNARII $^{-/-}$ou IFN $\gamma^{-/-}$ou IL-17RA ${ }^{-/-}$ou IL-1R $1^{-/-}$et des anticorps neutralisants ces récepteurs ou cytokines ou cellules [1,2]. En revanche, l'appareil de lyse (constitué par la perforine ou TRAIL) n'intervient pas dans ces effets. De fait, l'étude des réponses immunitaires dans les ganglions drainants la tumeur montre que la chimiothérapie augmente la sécrétion d'IFN $\gamma$ par les lymphocytes spécifiques des antigènes tumoraux. De plus, les cellules mourantes (par contact avec les anthracyclines) deviennent immunogènes en absence d'adjuvant et induisent une réponse lymphocytaire T productrice d'IFN $\gamma$. De plus, l'analyse cinétique par cytofluorométrie des infiltrats tumoraux post-chimiothérapie met en évidence d'abord un recrutement de lymphocytes $\mathrm{T} \gamma \delta$ producteurs d'IL-17, puis l'accumulation de lymphocytes T CD8+ producteurs d'IFN $\gamma$ et à moindre niveau, de lymphocytes T CD4+ producteurs d'IFN $\gamma$. Enfin, le profil transcriptionnel des gènes intratumoraux analysé 5-7 jours post-chimiothérapie révèle une cascade cytokinique commençant par ceux de la famille de l'IL-17 puis de l'IFN $\gamma$ [2].

Quels cytotoxiques induisent une réponse immunitaire in vivo ?

Une recherche à large échelle aléatoire de tous les composés cytotoxiques a révélé que seules, certaines drogues comme les anthracyclines, le cyclophosphamide, l'oxaliplatine ainsi que les rayons $\mathrm{X}$, peuvent provoquer cet afflux de lymphocytes intratumoraux et une mort immunogène. Ces composés ont la propriété de déclencher les stress prémortem appropriés pour rendre la cellule tumorale «immunogène» (notamment le stress du réticulum endoplasmique et l'autophagie, voir ci-dessous).

\section{Stress du réticulum endoplasmique et immunogénicité de la mort}

Une des conditions majeures pour l'induction d'une réponse immunitaire antitumorale est la capacité de certaines cellules du système immunitaire inné à phagocyter les cellules tumorales, puis à apprêter et à présenter les antigènes tumoraux à des lymphocytes $\mathrm{T}$ naïfs. Notre équipe $\mathrm{a}$ contribué à mettre en évidence le premier signal indispensable à la reconnaissance des cellules tumorales mourantes par les CD. Le signal de phagocytose généré par les cellules tumorales exposées aux anthracyclines, à l'oxaliplatine ou aux radiations ionisantes est l'exposition, à la surface de la membrane plasmique, d'une protéine du réticulum endoplasmique, la calréticuline 
(CRT) [3, 4]. La CRT est retrouvée à la surface de cellules mourantes dans les heures suivant le stress chimiothérapeutique et est caractéristique de la mort cellulaire immunogène, constituant ainsi un des premiers régulateurs de l'effet immunoadjuvant de la mort cellulaire tumorale. Un de nos résultats les plus surprenants est qu'une chimiothérapie non immunogène comme l'étoposide ou la mitomycine $\mathrm{C}$ (incapable de provoquer la translocation de la calréticuline à la membrane) devient soudainement agent antitumoral très efficace lorsqu'elle est combinée à un agent pharmacologique provoquant l'exposition de la CRT (comme la tautomycine) ou combinée à la CRT recombinante injectée en intratumoral. Ces effets synergiques (étoposide+ CRT locale) disparaissent chez les souris immunodéficientes. [4].

L'exposition de la CRT à la membrane plasmique résulte d'une cascade d'évênements intracellulaires appelés «stress du réticulum endoplasmique » qui se décompose en trois étapes : la phosphorylation, par la kinase PERK, du facteur d'initiation de la traduction eucaryote eif2 $\alpha$, ou l'inhibition de la déphosphorylation de la phosphatase PP1/GADD34, suivi du clivage de BAP31 par la caspase 8 activée, puis de l'activation des facteurs pro-apoptotiques Bax et Bak, enfin du transport antérograde de la CRT vers l'appareil de Golgi où le transport de la CRT est effectué par des vésicules dépendant des SNARE [5].

\section{Signal d'inflammation aseptique et engagement de TLR4 pour la mort immunogène}

La phagocytose des corps apoptotiques exposant la CRT par les CD est nécessaire mais non suffisante pour expliquer l'immunogénicité de la mort cellulaire engendrée par les chimiothérapies. Un second signal d'apoptose tardive est aussi fondamental : le relargage d'un facteur nucléaire, HMGB1 (High mobility group box 1 protein), protéine se fixant à l'ADN n'appartenant pas au groupe des histones. L'HMGB1 est relarguée dans le milieu extracellulaire dans les phases tardives de l'apoptose ou nécrose, pour se fixer sur le récepteur TLR4 (Toll-Like Receptor 4, récepteur des lipopolysaccharides) et déclencher une réponse inflammatoire au niveau des CD (dépendante de l'adaptateur Myd88). La signalisation des CD via TLR4 conduit à une rétention du chargement phagocyté dans les endosomes tardifs, évitant une dégradation de ce contenu dans les lysosomes. Ainsi, l'apprêtement antigénique des CD compétentes pour la transduction de signal via TLR4 se fait de façon plus efficace qu'en l'absence de TLR4 ou Myd88. Le ligand de TLR4 est HMGB1 dans le contexte d'une chimiothérapie. Les cellules tumorales déficientes en HMGB1 perdent leur immunogénicité post-chimiothérapie. De plus, les patients porteurs d'un allèle muté de TLR4 (d'un polymorphisme génétique - Asp299Gly - au niveau de TLR4 provoquant une perte de fixation du LPS ou HMGB1 à TLR4) répondent moins efficacement à la chimiothérapie adjuvante dans le cancer du sein ou du colon [6, 7].

\section{Inflammasome et immunité antitumorale}

Nos travaux récents montrent que les modifications post-chimiothérapie engendrées par les stress premortem sont non seulement de nature biochimique/protéique (HMGB1/CRT) mais aussi de nature métabolique. En effet, le relargage d'ATP par la cellule tumorale mourante est également nécessaire pour l'activation des récepteurs purinergiques P2RX7 exprimés par les CD. L'activation des récepteurs P2RX7 déclenche une cascade d'évènements majeurs, comme l'assemblage de l'inflammasome Nlrp3 qui active la caspase 1 permettant la libération de la cytokine inflammatoire IL-1 $\beta$. L'IL-1 $\beta$ est une molécule co-activatrice des lymphocytes T cytotoxiques qui deviennent alors des sources importantes d'IFN $\gamma$. En l'absence d'un des 
éléments moléculaires de la plateforme de l'inflammasome (P2RX7, Nlrp3, ASC, caspase-1, IL1ß/IL-1R1), l'immunogénicité des cellules tumorales mourantes est affectée et l'efficacité de la chimiothérapie anti-tumorale diminue très fortement [1]. De plus, les patientes porteuses d'un cancer mammaire et traitées en adjuvant par anthracyclines répondent moins efficacement à la chimiothérapie si elles sont porteuses d'un allèle muté (polymorphisme génétique provoquant une perte de fonctionnalité) de P2RX7 [1].

Ainsi, nos travaux ont mis en évidence 3 signaux immunogènes majeurs : La CRT, exposée en surface des cellules mourantes ayant subit un stress du réticulum endoplasmique, l'HMGB-1, relargué par les cellules en nécrose tardive (post apoptose) et enfin l'ATP pour lequel les mécanismes conduisant à sa sécrétion post chimiothérapie immunogène restent inconnus. L'autophagie est un processus physiologique de survie cellulaire induit par la privation en nutriments du milieu, l'hypoxie, ou lors d'un stress métabolique. Ce processus consiste en une auto-digestion lysosomale des composés cellulaires tels que les macromolécules, les ribosomes ou les organelles (réticulum endoplasmique, appareil de Golgi et mitochondries). Ce processus de défense est aussi utilisé pour lutter contre des infections bactériennes on parle alors de xénophagie. L'auto- ou la xéno-phagie sont des processus reliés au stress du réticulum endoplasmique [8], pouvant être induits par des chimiothérapies immunogènes. C'est pourquoi nous avons étudié le rôle potentiel de l'autophagie dans le processus de mort immunogène. $\mathrm{Ce}$ manuscrit va détailler la nécessité d'un autre stress, l'autophagie pour assurer une mort tumorale immunogène, à travers la sécrétion d'ATP.

\section{Résultats}

Afin de déterminer le rôle de l'autophagie dans l'immunogénicité de la mort tumorale induite par la chimiothérapie, nous avons étudié l'émission des signaux immunogènes identifiés à ce jour par des lignées tumorales murines rendues incompétentes pour l'autophagie (en inactivant des gènes codant pour des protéines clés dans ce processus, notamment Atg5 et Atg7). Ces cellules seront nommées par la suite déficientes en autophagie, Atg5 ${ }^{\mathrm{KD}}$ ou Atg $7^{\mathrm{KD}}$. Ainsi, les cellules CT26 (lignée de carcinome colorectal) control, Atg $5^{\mathrm{KD}}$ ou Atg $7^{\mathrm{KD}}$, entrent en apoptose de façon similaire après traitement par chimiothérapie immunogène (Mitoxantrone, MTX ou Oxaliplatine, Oxa). De plus, ces cellules restent capables d'exposer la CRT à la membrane plasmique et relarguent HMGB-1. En revanche, la déficience en autophagie ne permet plus la sécrétion d'ATP. Ces données indiquent que l'induction de l'autophagie par la chimiothérapie est un processus essentiel à la sécrétion d'ATP.

L'injection sous cutanée, sans aucun adjuvant, de la lignée tumorale CT26 (compétente pour l'autophagie) traitée par chimiothérapie élicite une réponse immunitaire anti-tumorale protectrice, capable de prévenir la croissance de la même lignée, injectée vivante ultérieurement. Cette protection est médiée par les lymphocytes T producteurs d'IFN $\gamma$. Les cellules déficientes en autophagie ne sont quant à elles pas capables d'éliciter une telle réponse protectrice, se traduisant par l'absence de lymphocytes T producteurs de l'IFN $\gamma$. L'administration aux souris d'un inhibiteur des ecto-ATPases (enzymes dégradant l'ATP extracellulaire) tel que ARL67156 (ARL), est un moyen permettant d'augmenter artificiellement la concentration d'ATP extracellulaire et donc de compenser le défaut d'émission d'ATP par les cellules déficientes en autophagie. Lorsqu' ARL et les cellules déficientes en autophagie sont co-administrées, la 
protection vaccinale est restaurée. Cette restauration peut être bloquée par l'administration d'antagonistes aux récepteurs purinérgiques tels que l'oxy-ATP (ATP oxydé) ou la suramine, confirmant l'importance de la signalisation ATP/P2RX7. La reconnaissance de l'ATP par les CD via le récepteur purinérgique P2RX7 permet, en complément du signal TLR4 apporté par HMGB-1 [6], la production d'IL-1 $\beta$, essentielle à l'élaboration de la réponse anti-tumorale après chimiothérapie [1]. Ainsi, l'administration d'IL-1 $\beta$, conjointement aux cellules déficientes en autophagie restaure également l'immunogénicité de ces dernières. L'ensemble de ces données indique que l'induction d'autophagie par la chimiothérapie est essentielle dans un protocole prophylactique pour élaborer une réponse immunitaire anti-tumorale protectrice.

Lorsque des souris porteuses de tumeurs sont traitées par chimiothérapie immunogène, celles-ci voient leur croissance tumorale contrôlée au cours du temps, de façon dépendante du système immunitaire $[1,2,4,6,9,10]$. En effet, l'émission de signaux immunogènes et leur perception par le système immunitaire conduit au recrutement intra-tumorale de CD (CD11c+), au niveau des foci d'apoptoses (Caspase 3a+). Ceci est suivi du recrutement de lymphocytes $\mathrm{T}$ CD4+ et CD8+ anti-tumoraux, producteurs d'IFN $\gamma$ ainsi que de lymphocytes $\mathrm{T} \gamma \delta$, producteurs d'IL-17. L'ensemble de ces cellules joue un rôle clé dans l'élaboration de la réponse antitumorale après chimiothérapie immunogène $[1,2,4,6,9]$ (Figure 1). Nous avons ainsi étudié l'ensemble de ces paramètres après traitement en protocole thérapeutique de souris porteuses de tumeurs déficientes en autophagie. Tout d'abord, les tumeurs ainsi déficientes ne sont plus contrôlées in vivo après chimiothérapie. Ceci s'explique par l'absence de recrutement de CD dans la tumeur, ainsi qu'à un défaut d'élaboration d'une réponse immune adaptative efficace, en d'autres termes un défaut de réponse lymphocytaire T CD4+, CD8+ et TcR $\gamma \delta+$ (sur la base de la sécrétion d'IFN $\gamma$ et d'IL-17, respectivement). L'administration d'ARL à des souris porteuses de tumeurs déficientes en autophagie restaure le contrôle de la croissance tumorale in vivo. Ceci ce traduit par la restauration du recrutement intra-tumorale des CD CD11c+, suivit de l'induction de LT CD4+, CD8+ et de LT $\gamma \delta$, producteurs d'IFN $\gamma$ et d'IL-17 respectivement.

Collectivement, l'ensemble de nos données indique que l'induction d'autophagie par la chimiothérapie est essentielle à l'émission de l'ATP, l'un des 3 signaux immunogènes clés de la mort cellulaire, et donc à l'élaboration d'une réponse immunitaire anti-tumorale efficace.

\section{Discussion}

L'effet principal de la chimiothérapie est l'induction d'une cytotoxicité directe sur les cellules tumorales. Nos travaux ont mis en évidence qu'au-delà de la cytotoxicité directe, certaines chimiothérapies (telles que les anthracyclines ou l'oxaliplatine) ainsi que la radiothérapie induisent une mort tumorale immunogène caractérisée par l'émission de 3 signaux majeurs. La CRT, exposée à la surface des cellules mourantes suite au stress du réticulum endoplasmique et facilitant la phagocytose de ces dernières ; 1'HMGB-1, reconnu par le TLR4 et favorisant la présentation antigénique ainsi que la production de pro-IL1 $\beta$. Enfin, l'émission d'ATP, reconnu par les récepteurs purinérgiques, et notamment le P2RX7 au niveau des CD. Ceci permet l'activation de l'inflammasome culminant avec la synthèse d'IL-1 $\beta$. Les travaux présentés ici montrent que l'induction de l'autophagie par la chimiothérapie est un processus capital pour le relarguage de l'ATP. Ce troisième signal permet le recrutement des CD au niveau du lit tumoral et l'élaboration d'une réponse immunitaire adaptative anti-tumorale caractérisée par la présence de LT CD4+ et CD8+ producteurs d'IFN $\gamma$. 
L'autophagie est un processus dont le rôle est controversé dans les cancers [11]. Certaines études montrent notamment que les cellules tumorales expriment plus faiblement les protéines impliquées dans le processus d'autophagie (LC3, Beclin-1). Une perte de fonction du gène codant pour beclin-1 (BECN1) favorise la tumorigénèse [12] alors qu'une surexpression de beclin-1 l'inhibe [13]suggérant que la déficience en autophagie joue un rôle dans le processus de transformation maligne. De plus, le gène BECN1 est muté dans $40 \%$ des cancers de la prostate, $50 \%$ des cancers du sein et dans $75 \%$ des cancers ovariens $[13,14]$. Le niveau d'expression de BECN1 est réduit dans d'autres cancers comme le cancer du côlon [15], du cerveau [16], et les carcinomes hépatocellulaires [17]. Ainsi, l'autophagie peut-elle être considéré comme un processus « suppresseur de tumeur » ou anti-oncogénique.

A l'inverse, l'autophagie est initialement un mécanisme assurant la survie cellulaire lors de stresses environnementaux ou cellulaires (privation en nutriments, hypoxie, stress thérapeutique) et confère une certaine résistance aux thérapies antinéoplasiques [18]. Des études récentes ont en effet montrées que l'inhibition de l'autophagie rendait les cellules tumorales plus sensibles aux agents cytotoxiques ainsi qu'à l'hormonothérapie et à la radiothérapie [19-24]. A l'inverse, l'induction de l'autophagie dans certains cancers résistants à l'apoptose, pourrait être une réelle solution thérapeutique afin de restaurer une mort tumorale [25-28].

Notre travail ne constitue aucunement une recommandation quant à l'induction ou l'inhibition de l'autophagie dans le traitement des cancers. Au-delà de l'aspect purement pro- et anti-tumorale de l'autophagie, nous avons identifié ce processus comme étant majeur après traitement par chimiothérapie immunogène car il conduit à la production d'ATP, l'un des signaux essentiel caractérisant la mort tumorale immunogène. Ainsi les patients ayant un cancer présentant une déficience en autophagie pourraient certainement bénéficier de stratégies pharmacologiques visant à compenser les défauts d'émission de signaux immunogènes (Figure 2), dans ce cas l'émission d'ATP. Ainsi, au-delà du développement d'agents pouvant induire l'autophagie [29], l'élévation de la concentration locale d'ATP par l'administration d'inhibiteurs d'apyrase, peut-elle constituer une stratégie thérapeutique compensatoire.

\section{Conclusion}

Les résultats exposés dans ce travail soulignent le rôle majeur joué par le système immunitaire dans le traitement des pathologies tumorales par la chimiothérapie ou les nouveaux traitements ciblés. Compte tenu de ces éléments, il est certain que les futures stratégies thérapeutiques anticancéreuses, pour être optimales, devront prendre en compte non seulement les caractéristiques du produit, celles de la tumeur, mais aussi de l'hôte :

- Il serait important de déterminer les caractéristiques immunologiques intrinsèques des composés cytotoxiques par des méthodes d'analyse à haut débit. Ces informations permettront de mieux sélectionner les produits d'amont de l'industrie pharmaceutique et de mieux entrevoir leurs combinaisons avec des immunothérapies.

- Il paraît possible de prédéterminer les caractéristiques immunologiques intrinsèques des cellules tumorales. Des tumeurs déficientes pour les molécules du stress du réticulum endoplasmique et /ou pour les mécanismes d'autophagie (ce qui est le cas de la plupart des tumeurs avancées), n'auront pas la capacité de relarguer les facteurs de danger nécessaires pour la mort immunogène. Une connaissance précise de leur capacité à relarguer HMGB1, à exposer la calréticuline à la membrane plasmique, à exposer les HSPs, à exercer l'agrégation des autophagosomes est 
envisageable par immunohistochimie. Ces éléments permettront de fournir des solutions d'immuno/chimiothérapies ciblées (compensatoires) et adaptées. Ainsi un déficit en autophagie se compense-t-il par un inhibiteur des ecto-ATPases.

- La connaissance des caractéristiques immunogénétiques [30] des patients permettra de déterminer les polymorphismes et variations déterminantes pour la réponse thérapeutique et l'évolution, comme le polymorphisme de TLR4 dans le cancer du sein, de l'IL-10 [31] dans le lymphome, de l'IL-18 dans le cancer de l'ovaire [32], ou du FcyRIIIA dans la réponse aux anticorps monoclonaux [33].

- L'existence de mécanismes de tolérance ou d'immunosuppression liés à la tumeur (Lymphocytes T régulateurs, cellules dendritiques myéloïdes immunosuppressives ou tolérogènes, sécrétion de TGF $\beta$, d'arginase, d'IL-10, de CTLA4) devra être supprimée par l'utilisation d'anticorps neutralisants, par des immunostimulants, ou par des stratégies de déplétion lymphocytaire transitoire

\section{Remerciements}

Nous tenons à remercier les soutiens financiers indispensables à la réalisation de ce travail : L'INSERM, La Ligue contre le Cancer, l'ARC, la FRM, l'AIRC, l'INCa et le Cancéropole Ile de France.

\section{Bibliographie}

[1]Ghiringhelli, F., L. Apetoh, A. Tesniere, et al. - Activation of the NLRP3 inflammasome in dendritic cells induces IL-1beta-dependent adaptive immunity against tumors. Nat Med, 2009. 15(10): p. 1170-8.

[2]Ma, Y., L. Aymeric, C. Locher, et al. - Contribution of IL-17-producing gamma delta T cells to the efficacy of anticancer chemotherapy. J Exp Med, 2011. 208(3): p. 491-503.

[3]Gardai, S.J., K.A. McPhillips, S.C. Frasch, et al. - Cell-surface calreticulin initiates clearance of viable or apoptotic cells through trans-activation of LRP on the phagocyte. Cell, 2005. 123(2): p. 321-34.

[4]Obeid, M., A. Tesniere, F. Ghiringhelli, et al. - Calreticulin exposure dictates the immunogenicity of cancer cell death. Nat Med, 2007. 13(1): p. 54-61.

[5]West, M.A., R.P. Wallin, S.P. Matthews, et al. - Enhanced dendritic cell antigen capture via toll-like receptor-induced actin remodeling. Science, 2004. 305(5687): p. 1153-7.

[6]Apetoh, L., F. Ghiringhelli, A. Tesniere, et al. - Toll-like receptor 4-dependent contribution of the immune system to anticancer chemotherapy and radiotherapy. Nat Med, 2007. 13(9): p. 1050-9.

[7]Tesniere, A., F. Schlemmer, V. Boige, et al. - Immunogenic death of colon cancer cells treated with oxaliplatin. Oncogene, 2010. 29(4): p. 482-91.

[8]Levine, B., N. Mizushima, and H.W. Virgin - Autophagy in immunity and inflammation. Nature, 2011. 469(7330): p. 323-35.

[9]Casares, N., M.O. Pequignot, A. Tesniere, et al. - Caspase-dependent immunogenicity of doxorubicininduced tumor cell death. J Exp Med, 2005. 202(12): p. 1691-701.

[10]Michaud, M., I. Martins, A.Q. Sukkurwala, et al. - Autophagy-dependent anticancer immune responses induced by chemotherapeutic agents in mice. Science, 2011. 334(6062): p. 1573-7.

[11]Dalby, K.N., I. Tekedereli, G. Lopez-Berestein, et al. - Targeting the prodeath and prosurvival functions of autophagy as novel therapeutic strategies in cancer. Autophagy, 2010. 6(3): p. 322-9.

[12]Qu, X., J. Yu, G. Bhagat, et al. - Promotion of tumorigenesis by heterozygous disruption of the beclin 1 autophagy gene. J Clin Invest, 2003. 112(12): p. 1809-20. 
[13]Liang, X.H., S. Jackson, M. Seaman, et al. - Induction of autophagy and inhibition of tumorigenesis by beclin 1. Nature, 1999. 402(6762): p. 672-6.

[14]Aita, V.M., X.H. Liang, V.V. Murty, et al. - Cloning and genomic organization of beclin 1, a candidate tumor suppressor gene on chromosome 17q21. Genomics, 1999. 59(1): p. 59-65.

[15]Koneri, K., T. Goi, Y. Hirono, et al. - Beclin 1 gene inhibits tumor growth in colon cancer cell lines. Anticancer Res, 2007. 27(3B): p. 1453-7.

[16]Miracco, C., E. Cosci, G. Oliveri, et al. - Protein and mRNA expression of autophagy gene Beclin 1 in human brain tumours. Int J Oncol, 2007. 30(2): p. 429-36.

[17]Daniel, F., A. Legrand, D. Pessayre, et al. - Beclin 1 mRNA strongly correlates with Bcl-XLmRNA expression in human hepatocellular carcinoma. Cancer Invest, 2007. 25(4): p. 226-31.

[18]Kondo, Y., T. Kanzawa, R. Sawaya, et al. - The role of autophagy in cancer development and response to therapy. Nat Rev Cancer, 2005. 5(9): p. 726-34.

[19]Amaravadi, R.K., D. Yu, J.J. Lum, et al. - Autophagy inhibition enhances therapy-induced apoptosis in a Myc-induced model of lymphoma. J Clin Invest, 2007. 117(2): p. 326-36.

[20]Apel, A., I. Herr, H. Schwarz, et al. - Blocked autophagy sensitizes resistant carcinoma cells to radiation therapy. Cancer Res, 2008. 68(5): p. 1485-94.

[21]Boya, P., R.A. Gonzalez-Polo, N. Casares, et al. - Inhibition of macroautophagy triggers apoptosis. Mol Cell Biol, 2005. 25(3): p. 1025-40.

[22]Kanzawa, T., I.M. Germano, T. Komata, et al. - Role of autophagy in temozolomide-induced cytotoxicity for malignant glioma cells. Cell Death Differ, 2004. 11(4): p. 448-57.

[23]Paglin, S., T. Hollister, T. Delohery, et al. - A novel response of cancer cells to radiation involves autophagy and formation of acidic vesicles. Cancer Res, 2001. 61(2): p. 439-44.

[24]Qadir, M.A., B. Kwok, W.H. Dragowska, et al. - Macroautophagy inhibition sensitizes tamoxifenresistant breast cancer cells and enhances mitochondrial depolarization. Breast Cancer Res Treat, 2008. 112(3): p. 389-403.

[25]Akar, U., B. Ozpolat, K. Mehta, et al. - Tissue transglutaminase inhibits autophagy in pancreatic cancer cells. Mol Cancer Res, 2007. 5(3): p. 241-9.

[26]Moretti, L., A. Attia, K.W. Kim, et al. - Crosstalk between Bak/Bax and mTOR signaling regulates radiation-induced autophagy. Autophagy, 2007. 3(2): p. 142-4.

[27]Shimizu, S., T. Kanaseki, N. Mizushima, et al. - Role of Bcl-2 family proteins in a non-apoptotic programmed cell death dependent on autophagy genes. Nat Cell Biol, 2004. 6(12): p. 1221-8.

[28]Xue, L., G.C. Fletcher, and A.M. Tolkovsky - Autophagy is activated by apoptotic signalling in sympathetic neurons: an alternative mechanism of death execution. Mol Cell Neurosci, 1999. 14(3): p. 180-98.

[29]Fleming, A., T. Noda, T. Yoshimori, et al. - Chemical modulators of autophagy as biological probes and potential therapeutics. Nat Chem Biol, 2011. 7(1): p. 9-17.

[30]Tsung, A., R. Sahai, H. Tanaka, et al. - The nuclear factor HMGB1 mediates hepatic injury after murine liver ischemia-reperfusion. J Exp Med, 2005. 201(7): p. 1135-43.

[31]Lech-Maranda, E., L. Baseggio, J. Bienvenu, et al. - Interleukin-10 gene promoter polymorphisms influence the clinical outcome of diffuse large B-cell lymphoma. Blood, 2004. 103(9): p. 3529-34.

[32]Bushley, A.W., R. Ferrell, K. McDuffie, et al. - Polymorphisms of interleukin (IL)-1alpha, IL-1beta, IL-6, IL-10, and IL-18 and the risk of ovarian cancer. Gynecol Oncol, 2004. 95(3): p. 672-9.

[33]Nimmerjahn, F. and J.V. Ravetch - Antibodies, Fc receptors and cancer. Curr Opin Immunol, 2007. 19(2): p. 239-45. 


\section{Figures et légendes}

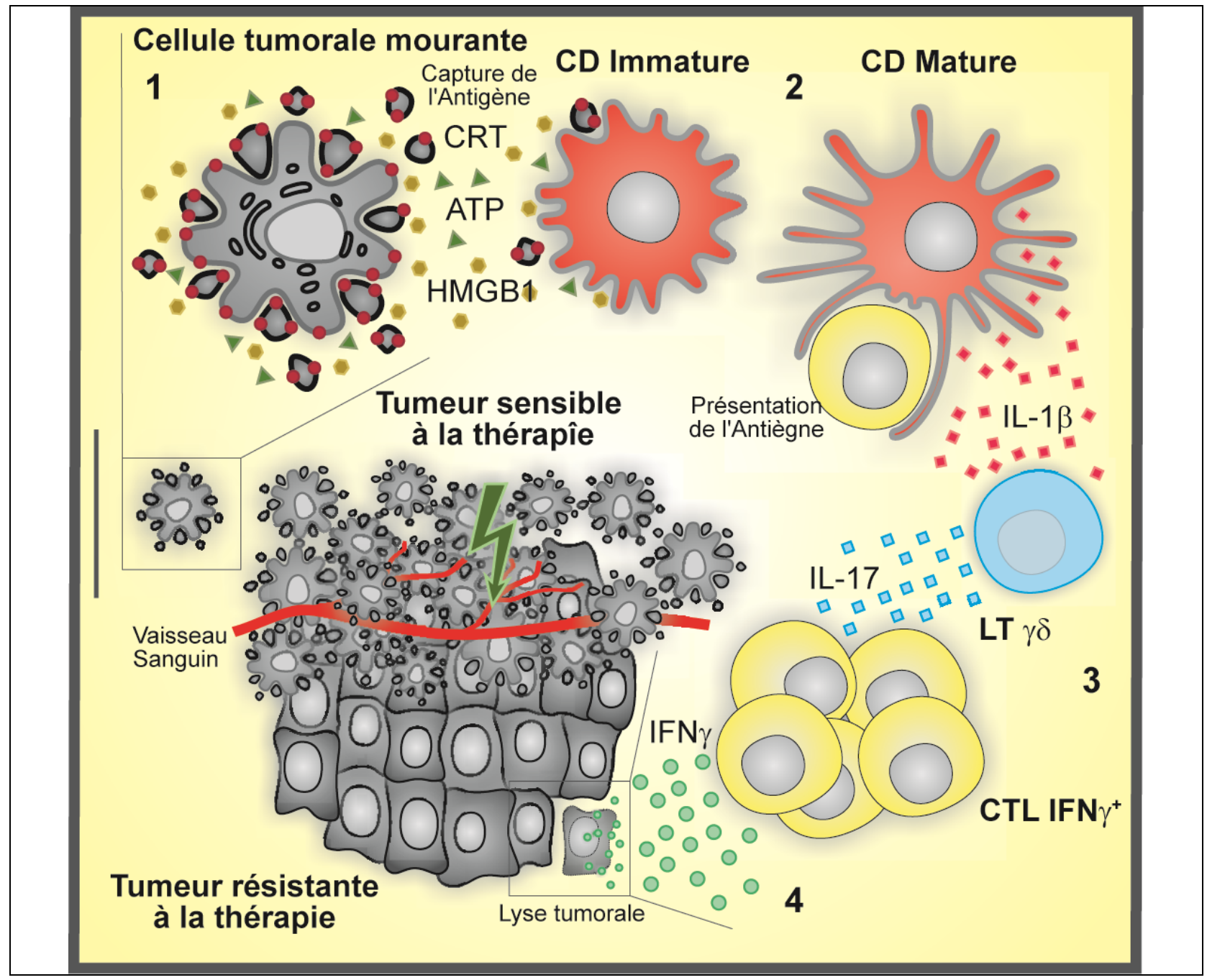

Figure 1: Orchestration précise de la réponse lymphocytaire $T$ après la mort tumorale immunogène 1 - Après traitement par chimiothérapie immunogène ou radiothérapie, les cellules tumorales émettent mourantes émettent les signaux caractéristiques de la mort immunogène, l'exposition de la CRT à la surface (suite à la réponse au stress du réticulum endoplasmique), le relarguage d'HMGB-1 (au cours de la mort cellulaire) et de l'ATP (suite à l'autophagie). 2- Ceci conduit à la phagocytose du matériel tumorale par les cellules dendritiques (CD) ainsi qu'à leur maturation. Les $\mathrm{CD}$ présentent alors les antigènes tumoraux aux lymphocytes $\mathrm{T}$ et sécrètent de l'IL-1 $\beta .3$ - Cette cytokine va activer les lymphocytes $\mathrm{T} \gamma \delta$ producteurs d'IL-17, contribuer à polariser les LT CD8+ en lymphocytes T cytotoxiques (CTL) producteurs d'IFN $\gamma .4$ - Les CTL, effecteurs terminaux de la réponse anti-tumorale vont massivement infiltrer la tumeur et éliminer les cellules tumorales résiduelles. 


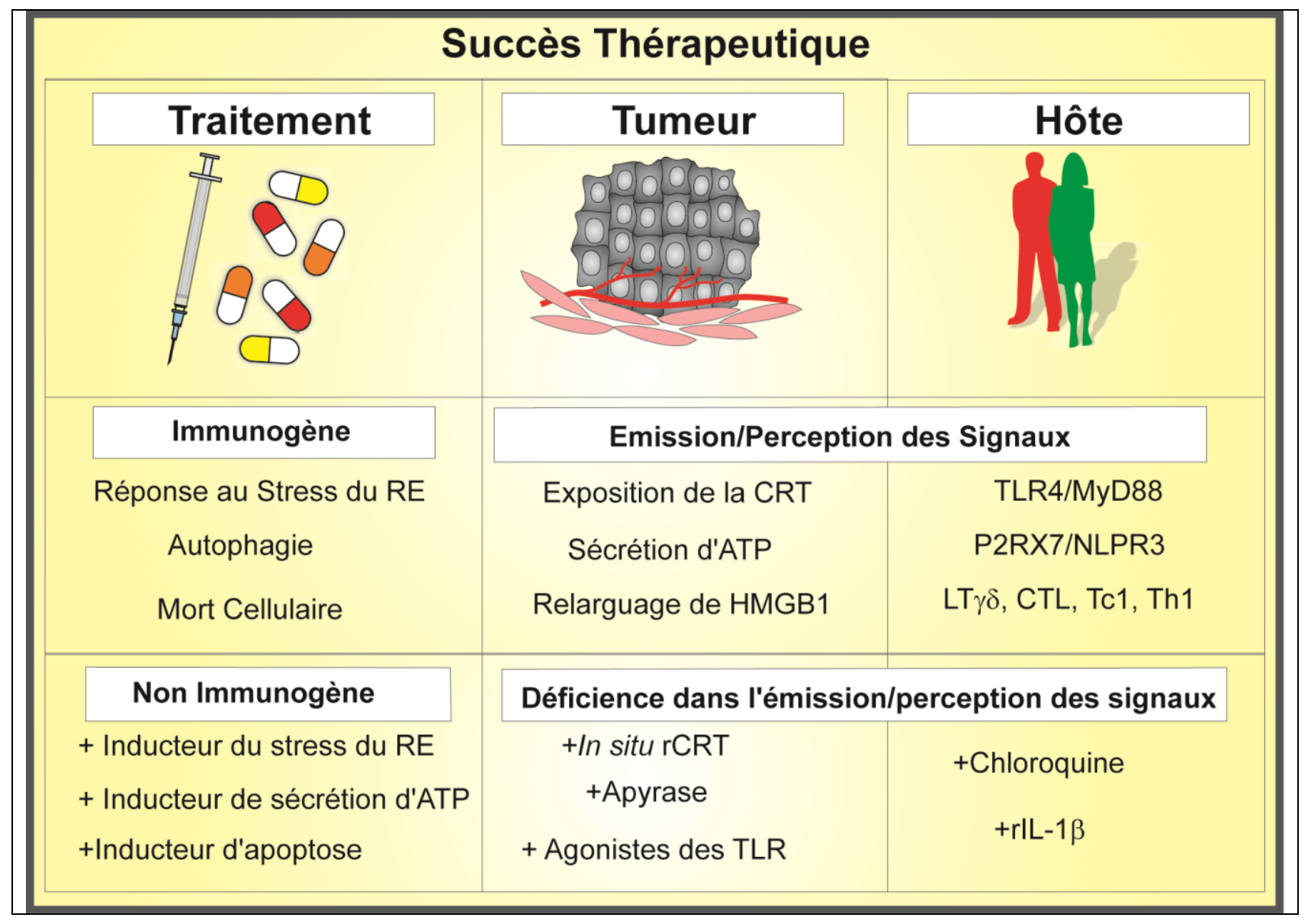

Figure 2: Vue schématique des traitements anti-cancéreux personnalisés:

Le succès thérapeutique dépend des propriétés intrinsèques du traitement, de la tumeur et de l'hôte (le patient). Tout d'abord le traitement doit induire une mort immunogène, et pour cela induire un stress du RE ainsi que l'autophagie et enfin la mort de la cellule cible. Ceci permet l'émission des signaux immunogènes par la tumeur (CRT, ATO, HMGB-1) sont perçus par l'immunité de l'hôte via des récepteurs (TLR4, P2RX7, NLRP3). Cette signalisation permet le recrutement et l'activation des cellules effectrices (CTL, LT $\gamma \delta)$ et à l'élimination des cellules tumorales. Pour garantir le succès thérapeutique, il faudrait développer des stratégies thérapeutiques compensatoires personnalisées. Tout d'abord au niveau du traitement (combiner une chimiothérapie non immunogène avec un inducteur de stress du RE par exemple). Il faudrait déterminer les défauts éventuels d'émission d'un (ou plusieurs) des signaux immunogènes par la tumeur du patient. Par exemple compenser un défaut d'exposition de la CRT au moyen de CRT recombinante, ou encore compenser un défaut d'émission d'HMGB-1 par l'ajout d'un agoniste du TLR4. De manière similaire, les mutations « perte de fonction » des récepteurs clés impliqués dans la reconnaissance des signaux immunogènes doivent être ciblées et compensées par exemple en ciblant une voie TLR alternative au TLR4, ou à l'aide de cytokines appropriées. 